\title{
MEASUREMENT OF THE ANISOTROPY IN PERMALLOY
}

Kees J. Eijkelt, Twente University of Technology

P. 0. Box 217, 7500 AE Enschede, The Netherlands

+ The Netherlands Technology Foundation (STW)

\section{Abstract}

In many applications of permalloy, detailed information on the uniaxial anisotropy is needed. A measurement system is described, which accurately determines the anisotropy field $\mathrm{H}_{k}$ and the orientation of the easyaxis in a permalloy film or in any material showing magnetization induced resistance anisotropy. An accuracy of $0.1 \%$ in $H_{k}$ and $0.1^{\circ}$ in easy-axis orientation is reached in permalloy when the applied fieldstrength is $2.5 \mathrm{H}_{\mathrm{k}}$. Deviations from the StonerWohlfarth single domain model can be recognized after the analysis of the measurement. Four electrical contacts have to be positioned on the permalloy in a planar-Hall configuration. We use aluminum contacts, realised in a standard lithographic process, providing a reference for the easy-axis orientation and an accurate relative orientation of the different planarHall elements that can be made out of a single film.

\section{Introduction}

A number of ways to determine the anisotropy fieldstrength and orientation in a permalloy film have been described. An overview of these methods is presented in [1]. The accuracy of many of these methods is strongly influenced by the magnetization behavior in a nonsaturated film. Kempter and Hoffmann [2] and Kneer [1] proposed methods that overcome these problems. Accurate values for the anisotropy fieldstrength $\mathrm{H}_{\mathrm{k}}$ are derived. However there is no indication of the quality of the film (anisotropy dispersion, magnetic form effects). Measurements in higher fields can use ferromagnetic resonance or a torque magnetometer, but complicated measurement equipment is required [3]. Accurate measurement of the easy-axis orientation is only possible with the torque magnetometer technique.

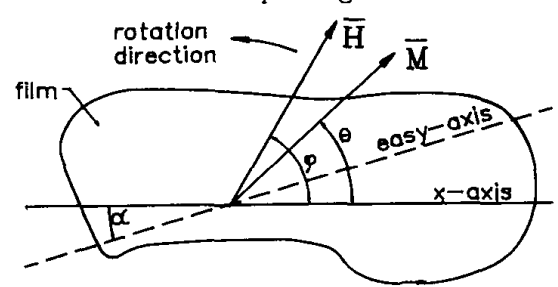

Fig 1: Anisotropy in a permalloy film.

With the possibility, introduced with the computer, of recording and analyzing a great number of data, other techniques, previously inconceivable because of the amount of work involved, are gaining interest. Magnetically it is desirable to keep the film in a saturated state. The method proposed uses a constant in-plane field of 2 to 20 times $H_{k}$, slowly rotating with respect to the film (see figure 1). The field direction is determined by measurement of the angle $\varphi$. The magnetization induces a resistance anisotropy in the material [4]. When four electrical contacts are positioned on the film in a planar-Hall configuration [5] (see figure 2) we find:

$\mathrm{V}_{\mathrm{ph}}=\mathrm{I} \frac{\Delta \rho}{2 t} \sin (2 \Theta)$

with $t$ the film thickness and $\Delta p$ the resistivity anisotropy. The shape of the film may be arbitrary. The current contacts should be positioned at some distance from the voltage contacts to prevent short-circuiting of the planar-Hall effect.

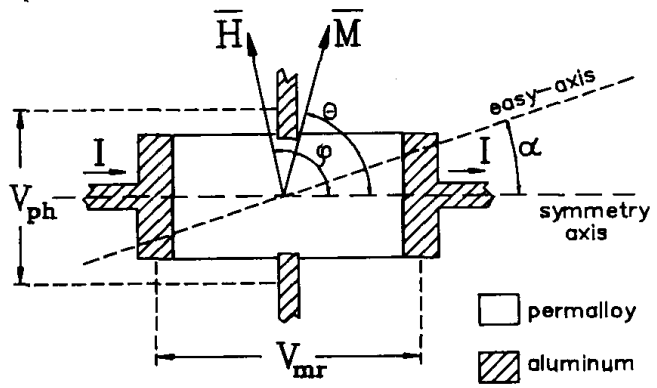

Fig 2: A planar-Hall configuration.

In (1) the voltage contacts are considered to be point contacts, while the current at a point in the film is assumed to be constant over the film thickness. The angle $\Theta$, determining the magnetization direction, can be calculated from $V_{p h}$. The electrical contacts give a geometric reference for the anisotropy orientation, the symmetry axis (see figure 2). When modern lithographic techniques are used, accurate positioning of contacts is possible.

The angle $\varphi$ - $\theta$ between magnetization and field is determined for several field directions. It will follow the Stoner-Wohlfarth single domain model [6];

$\sin (\varphi-\Theta)=\frac{H_{k}}{2 H} \sin 2(\Theta-\alpha)$

Comparing the behavior of $\varphi-\theta$ with the Stoner-Wohlfarth model, the ratio $H_{k} / H$ and the angle between the easyaxis and the symmetry axis of the element, $\alpha$, can be calculated.

\section{The Measurement Equipment}

Figure 3 shows the measurement equipment. The permalloy film is mounted on a rotation stage (accuracy $0.01^{\circ}$ ). A pair of Helmholtz coils provides a homogeneous magnetic field $\overrightarrow{\mathrm{H}}$ of maximum $6000 \mathrm{~A} / \mathrm{m}$. To limit the dissipation in the $f i l m$ and to cancel out offset and drift in the measuring circuit, a lock-in amplifier (PAR model 128A) is used for amplification of the output signal $V_{p h}$ of the planar-Hall element.

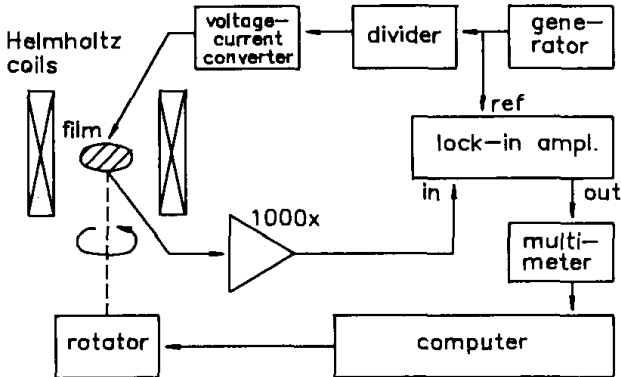

Fig 3: The measurement equipment.

A $500 \mathrm{~Hz}$ sine generator provides a $1 \mathrm{~V}$ RMS reference signal for the lock-in amplifier. This voltage is divided with a dekavider DV54 (accuracy $10 \mathrm{ppm}$ ) and 
converted to a current, driving the planar-Hall element. The output signal from the planar-Hall element has high common-mode. An extra instrumentation amplifier with high dynamic CMRR is used to increase the common-mode rejection of the system. The output of the lock-in amplifier is connected with a high resolution digital multimeter. A computer drives the steppermotor and stores the readings from the multimeter. Calibra$t$ ion of the amplifying system showed a non-linearity of less than $0.01 \%$. Using (1) one finds that $\theta$ can be determined within $\pm 0.01 \%$ in $60 \%$ of its range and within $0.02^{\circ}$ in $85 \%$ of its range. The error in $\varphi$ depends on the rotation stage and is maximum $0.01^{\circ}$. The total error in $\varphi-\Theta$ is the sum of both errors. Dissipation was less than $50 \mu \mathrm{W}$ in all elements.

\section{The Analysis}

The analysis of the data is executed in two steps: First the magnetization direction $\Theta$ as a function of field direction $\varphi$ is calculated. In the second step, the anisotropy parameters are determined.

\section{First step:}

The signal voltage from a planar-Hall element is measured at 180 field directions, beginning at $\varphi=\beta$ and increasing $1^{\circ}$ at a time to $\varphi=\beta+179^{\circ}$. Usually the signal voltage is the sum of $V_{p h}$ and an offset voltage $V_{\text {off }}$, consisting of a small fraction $f$ of the voltage across the current contacts of the element, $V_{m r}$ (see figure 2) This voltage can be described by [5]:

$\mathrm{V}_{\text {off }}=f \mathrm{~V}_{\mathrm{mr}}=f \mathrm{I}\left(\mathrm{k} \rho-\mathrm{k} \Delta \rho \sin ^{2} \Theta\right)$

with $\mathbf{k}$ a geometric factor, depending on the shape of the element, and $\rho$ the resistivity of the permalloy. Combining (1) and (3) and introducing A, B and $\delta$ as described below, the signal voltage from the planarHall element is found to be:

$V_{\text {sig }}=A+B \sin (20+\delta)$

$A=f k \rho\left(1-\frac{\Delta \rho}{2 \rho}\right) \quad B=\frac{\Delta \rho}{2 t} \sqrt{1+(f k t)^{2}}$

$\delta=\arctan \left(\frac{A}{\sqrt{\left(1-\frac{2 \rho}{\Delta \rho}\right)^{2} B^{2}-A^{2}}}\right)$

$A$ and $B$ are the offset and amplitude of $V_{s i}$ The phase-shift $\delta$ in $V_{s i g}$, caused by the offset voltage $V_{o r f}$, can be determined directly from $A$ and $B$ when the magnetoresistance ratio $\Delta \rho / \rho$ is known.

Using (4) the behavior of $\sin (2 \theta+\delta)$ versus rotation stage position is found and $\Theta$ can be calculated for each position. To relate the position of the rotation stage to the field angle $\varphi$, the value of $\varphi$ at the start of the measurement, $\beta$, has to be determined. Assuming that the Stoner-Wohlfarth single domain model is valid, one can show that $\beta$ can be derived from the two rotation stage positions $\varphi_{1}$ and $\varphi_{2}$ at which $\sin (2 \Theta+\delta)$ has positive and negative zero-crossings respectively:

$\beta=\frac{1.5 \pi-\varphi_{1}-\varphi_{2}-\delta}{2} \quad 0 \leq \beta<\frac{\pi}{2}$

With the parameters A, B, $\delta$ and $\beta, \varphi$ - is calculated as a function of the field angle $\varphi$.

\section{Second step:}

The amplitude of $\varphi-\Theta$ versus $\varphi$ directly determines the ratio $\mathrm{H}_{\mathrm{k}} / \mathrm{H}$ through:
$\frac{\mathrm{H}_{\mathrm{k}}}{\mathrm{H}}=2 \sin (\varphi-\Theta)_{\max }$

Furthermore, $\varphi$ equals $\alpha$ at the positive zero-crossing of $\varphi-\Theta$, so:

$$
\left.\begin{array}{c}
\varphi-\Theta=0 \\
\frac{d(\varphi-\Theta)}{d \varphi}>0
\end{array}\right\} \quad \varphi=\alpha
$$

As shown above, the measured values of $\Theta$, and therefore of $\varphi-\Theta$, have lower accuracy in some parts of their range. To reduce the influence of this effect on the determination of $\alpha$ and $\mathrm{H}_{\mathbf{k}} / \mathrm{H}$, theoretical values for $\varphi-\theta$ are calculated with (2) using the results of (6) and (7), and are fitted on the measured values, adjusting $\alpha$ and $\mathrm{H}_{\mathrm{k}} / \mathrm{H}$ for a closest fit.

A worst case calculation for our measurement equipment and analysis program shows a maximum error in $\alpha$ of less than $0.1^{\circ}$ and in $\mathrm{H}_{\mathrm{k}} / \mathrm{H}$ of less than $0.1 \%$ in a perfect single domain for $\mathrm{H} \approx 2.5 \mathrm{H}_{\mathrm{k}}$. Lower fieldstrengths will in principle give lower errors, but experiments show, that deviations from the single domain model occur, caused by an increased influence of form effects and anisotropy dispersion.

\section{Preparation Techniques}

The permalloy films are deposited on silicon wafers with $0.5 \mu \mathrm{m} \mathrm{SiO}_{2}$ on the surface. First, a contact pattern in aluminum is realised by wet chemical etching of a $0.5 \mu \mathrm{m}$ thick layer of evaporated aluminum. Then a $50 \mathrm{~nm} \mathrm{NiFe}$ film is RF-sputtered in a Leybold Heraeus Z400 sputter unit (voltage $800 \mathrm{~V}$, bias voltage $80 \mathrm{~V}$ ) in an $\mathrm{Ar}$ atmosphere $\left(2.510^{-2}\right.$ mbar $)$. The target consists of $82 \%$ nickel and $18 \%$ iron. A bias field of about 2000 $\mathrm{A} / \mathrm{m}$ is used to direct the easy-axis orientation. Wet chemical etching of the permalloy gives the planar-Hall elements their final shape. The wafers are broken and the different elements are mounted on epoxy substrates. Copper soldering pads on the epoxy substrate are used for the connecting wires. Bonding wires connect the soldering pads with the element.

\section{Measurement Results and Discussion}

Figure $4 a$ shows the measurement result of a typical film. To eliminate the influence of magnetic form effects on the magnetization in the permalloy, a circular shape was chosen. The diameter of the circle is $10 \mathrm{~mm}$. The crosses represent the measured values of $(\varphi-B)$ while the solid line represents the theoretical curve, fitted to the measurements. For clarity of the plot, not all 180 measurement points are shown.

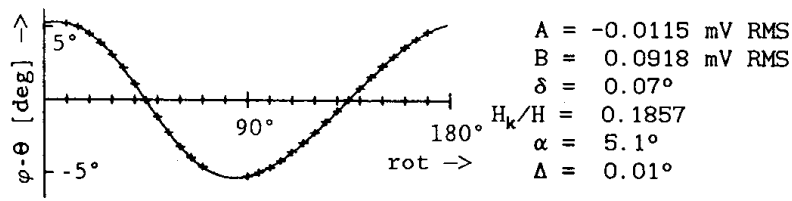

Fig 4a: $\varphi-\Theta$ vs rotation stage position for a typical film, $H=2000 \mathrm{~A} / \mathrm{m}, I=1.5 \mathrm{~mA} \mathrm{RMS}$.

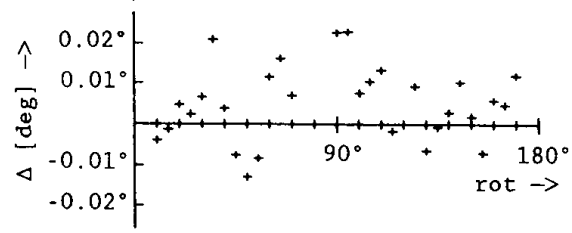

Fig 4b: Deviation from the model. 
Figure $4 \mathrm{~b}$ shows the difference between the theoretical curve and the measured values of $(\varphi-\Theta)$. The magnetization in the film follows the single domain model well within the measurement accuracy. $\Delta$ is the RMS value of the difference between measurement and model and is used as an indication for the quality of the film. Plots like figure $4 \mathrm{~b}$ give information about the quality of the measurement and the film. Errors caused by jamming of the rotation stage, torque through connecting wires, temperature change during the measurement etc. can readily be recognized. Furthermore, if the single domain model is not valid, the form of this plot can reveal the origin of the deviations.

In general, a permalloy film is not a perfect single domain. Dispersion in easy-axis orientation and anisotropy fieldstrength throughout the film and magnetic form effects will cause deviations from the single domain model.

The galvanomagnetic measurement technique determines an averaged magnetization direction for the film. This means, that local variations in magnetization direction can not be determined. However, when local variations in magnetization occur, the averaged magnetization direction measured will not follow the single domain model, and a close $f i t$ between measuring points and theoretical values for the $(\varphi-\Theta)$-plot can not be made. A plot like figure $4 b$ will now give information about the cause of these local variations in magnetization direction. The different possibilities will be discussed briefly.

When the film geometry provides a homogeneous demagnetizing field throughout the film, the permalloy will behave like a single domain with an effective anisotropy fieldstrength and orientation in which the intrinsic anisotropy and magnetic form effects are combined [7]. The effective anisotropy parameters are determined in the measurement.

Inhomogeneous demagnetizing fields cause a deviation from the single domain model. Figure 5 shows a measurement on a circular film ( $\varnothing 1 \mathrm{~mm})$ with small extensions of $250 \mu \mathrm{m}$ length leading to the aluminum contacts, $250 \mu \mathrm{m}$ wide for the current contacts and 100 $\mu \mathrm{m}$ wide for the voltage contacts. Deviations from the model are significant.

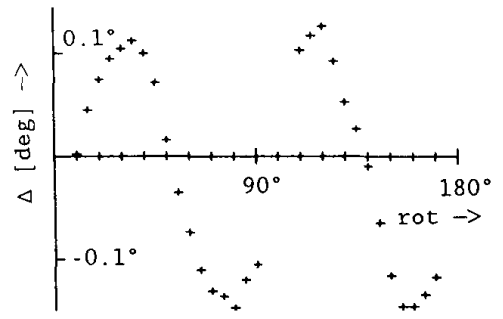

Fig 5: Deviation from the single domain model for a circular element with contact extensions, showing magnetic form effects.

Films with high angular dispersion in anisotropy can be produced when the bias field is removed during deposition of the film. A measurement on such a film has been performed (see figure 6). With the Crowther method [8], $\alpha_{90}$ was estimated to be $30^{\circ}$. Computer simulation of a film with $H_{k}=400 \mathrm{~A} / \mathrm{m}$ and similar angular dispersion gives comparable results. The measured anisotropy orientation is the average orientation in the film. $\mathrm{H}_{\mathbf{k}}$ is reduced with a factor of approximately 0.75 .

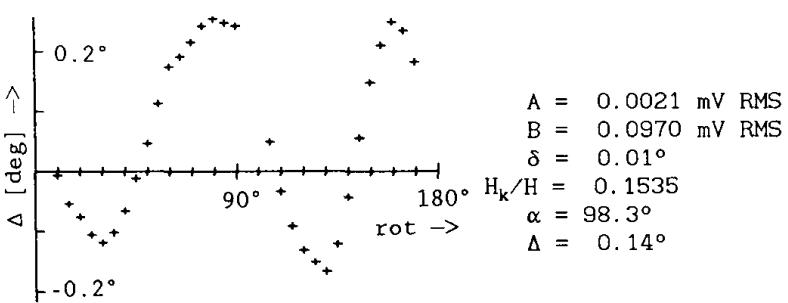

Fig 6: Deviation from the model for a high angular dispersion $\mathrm{film}, H=2000 \mathrm{~A} / \mathrm{m}, I=1.1 \mathrm{~mA} \mathrm{RMS}$.

Amplitude dispersion in anisotropy filmstrength only affects the measured $H_{k}$. An average value will be found. Computer simulations show, that the effect of amplitude dispersion on the measurement results is small, compared with other effects.

\section{Conclusions}

A measurement system is described which accurately determines the anisotropy fieldstrength $\mathrm{H}_{\mathrm{k}}$ and the orientation of the easy-axis in permalloy. Errors of less than $0.1 \%$ in $H_{\mathbf{k}}$ and 0.10 in easy-axis orientation are achievable when the applied fieldstrength is about $2.5 \mathrm{H}_{k}$, for the measurement equipment described. Deviations from the assumed single domain behavior can be recognized after analysis of the measurement. These deviations may be caused by inhomogeneous demagnetizing fields, or by dispersion in anisotropy fieldstrength and orientation.

Four electrical contacts have to be positioned on the film in a planar-Hall configuration. Non-magnetic prober pens may be used. On the other hand, the use of aluminum contacts, realised by standard lithographic techniques provides an accurate reference for the measured easy-axis orientation.

Measurements show good stability and reproducibility.

\section{Acknowledgments}

This work is supported by the Netherlands Technology Foundation (STW). The author would like to thank prof.dr.J.H.J. Fluitman, ir.P. de Haan and ir.R.M. de Ridder for helpful discussions.

\section{References}

[1] G. Kneer, The anisotropy field $H_{k}$ of thin ferromagnetic films, measured by magnetoresistive methods, IEEE Trans. Magn. MAG-2, pp. 747-750 (1966).

[2] K. Kempter and H. Hoffmann, An improved method for the determination of the anisotropy field of ferromagnetic thin films, Zeitschr. f, angew. Phys. 28, pp. 251-257 (1970).

[3] F.R. Soohoo, Magnetic Thin Films. New York: Harper, 1965, pp. $128-131$.

[4] T.R. McGuire and R. I. Potter, Anisotropic magnetoresistance in ferromagnetic 3d alloys, IEEE Trans. Magn. MAG-11, pp. 1018-1038 (1975).

[5] K.J. Eijkel and J.H. Fluitman, Angle detection based on the resistance anisotropy of permalloy, IEEE Trans. Magn. MAG-22, pp. 955-957 (1986).

[6] M. Prutton, Thin Ferromagnet ic Films. London: Butterworth, 1964, p. 55.

[7] J.H.J. Fluitman, The influence of sample geometry on the magnetoresistance of $\mathrm{Ni}-\mathrm{Fe}$ films, Thin Solid Films 16, pp. 269-276 (1973)

[8] T.S. Crowther, Angular and magnitude dispersion of the anisotropy in magnetic films, Journal of Applied Physics 34, pp. 580-587 (1963). 